Published in final edited form as:

Nat Neurosci. 2011 February ; 14(2): 163-164. doi:10.1038/nn.2724.

\title{
Amygdala Volume and Social Network Size in Humans
}

\author{
Kevin C. Bickart ${ }^{1}$, Christopher I. Wright ${ }^{2}$, Rebecca J. Dautoff ${ }^{2}$, Bradford C. Dickerson ${ }^{\star}, 2,3$, \\ and Lisa Feldman Barrett ${ }^{\star}, 2,4$ \\ ${ }^{1}$ Department of Anatomy and Neurobiology, Boston University School of Medicine \\ ${ }^{2}$ Psychiatric Neuroimaging Research Program and Martinos Center for Biomedical Imaging \\ ${ }^{3}$ Department of Neurology, Massachusetts General Hospital and Harvard Medical School \\ ${ }^{4}$ Department of Psychology, Northeastern University
}

\begin{abstract}
We demonstrated that amygdala volume (corrected for total intracranial volume) positively correlated with the size and complexity of social networks in adult humans ranging in age from 19 to 83 years. This relationship was specific to the amygdala as compared to other subcortical structures. An exploratory analysis of the entire cortical mantle also revealed an association between social network variables and cortical thickness in three cortical areas, two of which share dense connectivity with the amygdala. Amygdala volume was not related to other social variables such as life satisfaction or social support. These findings converge with data from functional neuroimaging and lesion neuropsychology indicating that the amygdala plays an important role in brain networks contributing to social behavior.
\end{abstract}

\section{Keywords}

Amygdala; Social Brain; Brain Evolution; Social Network Size; MRI

For many species, but particularly for primates, living in groups is a major adaptive advantage 1. But living in a social group also presents its own challenges. To get along while getting ahead, it is necessary to learn who is who, who is friend, and who is foe. It might be productive to form an alliance with certain group members in one context, but to outmaneuver them in another. The "social brain hypothesis" suggests that, evolutionarily, living in larger, more complex social groups selected for larger brain regions with a greater capacity for performing relevant computations2. Based on its central functional role3, 4 and anatomic position ${ }^{19}$ in the social brain, investigators have proposed that amygdala volume should be related to the size of social groups, in part because the size of a brain region is one indicator of its processing capacity5, 6 .

Users may view, print, copy, download and text and data- mine the content in such documents, for the purposes of academic research, subject always to the full Conditions of use: http://www.nature.com/authors/editorial_policies/license.html\#terms

Corresponding Author: Lisa Feldman Barrett, Ph.D., Department of Psychology, Northeastern University, 125 Nightingale Hall, 360 Huntington Avenue, Boston, MA 02115, Telephone: (617) 373-3076, Fax: (617) 373-8714, 1.barrett@ neu.edu.

The authors made equal contributions to this work. 
Comparative neuroanatomical studies in nonhuman primates strongly support a link between amygdala volume and social network size7 and social behavior8. Species characterized by larger social groups have a larger corticobasolateral (CBL) complex within the amygdala. The CBL complex conjointly expanded with evolutionarily newer cortex size and the lateral geniculate nucleus (LGN), particularly the layers of the LGN that project to the ventral stream visual system7. Taken together, these comparative findings suggest that a larger amygdala provides for the increased processing demands required by a complex social life.

In this study we examined whether amygdala volume varies with individual variation in the size and complexity of social groupings within a single primate species (humans). In 58 normal healthy adults ( 22 females; age $M=52.6, S D=21.2$, range=19-83 years), we examined social network size and complexity with 2 subscales of the Social Network Index [SNI9]. One SNI subscale (Number of People in Social Network) measured the total number of regular contacts that a person maintains, reflecting overall network size. A second subscale (Number of Embedded Networks) measured the number of different groups these contacts belonged to, reflecting network complexity. Despite the fact that the two social network variables displayed a strong correlation within the present sample $(\mathrm{r}=.86, p<.001)$, we opted to consider their separate relation to amygdala and hippocampal volumes (for more details, see Supplementary Results online).

To assess amygdala (and, as a control region, hippocampal) volume, we performed quantitative morphometric analysis of T1-weighted MRI data using an automated segmentation and probabilistic region-of-interest (ROI) labeling technique (FreeSurfer, http://surfer.nmr.mgh.harvard.edu). For methodological details, see Supplementary Methods online. To adjust for differences in head size, amygdala and hippocampal volumes were divided by total intracranial volume as performed previously 10,11 .

Linear regression analyses revealed that individuals with larger and more complex social networks had larger amygdala volumes (see Fig. 1). These relationships held when controlling for the age of the participant (because older individuals have, on average, smaller amygdala volumes than do younger individuals; Table 1). These relationships held when left and right amygdala volumes were analyzed separately (see Table 1), indicating no lateralization of the effect.

To assess discriminant validity, we performed a linear regression using right and left hippocampal volumes (corrected for total intracranial volume) as independent variables and social network size and complexity as dependent variables while controlling for age (because hippocampal volume typically diminishes with age). For the whole group, these analyses revealed no significant relationship between hippocampal volume and either social network variable (see Table 1). For the young and older subgroups, linear regressions only revealed a significant relationship for older participants between left hippocampal volume and social network complexity (see Table 1). Because hippocampal and amygdala volumes were themselves strongly correlated (Left: $\mathrm{r}=0.831, p<0.001$; Right: $\mathrm{r}=0.727, p<0.001$; combined: $\mathrm{r}=0.815, p<0.001$ ), we conducted hierarchical linear regressions using amygdala and hippocampal volumes (corrected for total intracranial volume) as independent variables and social network characteristics as dependent variables. Increased amygdala 
volume remained significant when controlling for hippocampal volume (see Supplementary Table 1 online).

To further investigate the specificity of the relationship between amygdala volume and social network characteristics, we conducted an exploratory analysis assessing the relationship between social network variables and all other subcortical volumes segmented by Freesurfer. Linear regressions revealed that none of the additional subcortical regions significantly correlated with either social network variable when controlling for age and correcting for multiple comparisons. For more details, see Supplementary Methods and Results online.

Also supporting the discriminant validity of our primary finding, we found that amygdala volume did not relate to other measures of social functioning such as perceived social support12, 13 and life satisfaction14 (r's ranged from -.26 to $.27, p<.15$ to $p<.98$ ). For more details about these measures, see Supplementary Methods online.

Finally, to explore the association between social network variables and cortical thickness throughout the cerebral cortex, we conducted a whole brain surface-based analysis (see Supplementary Methods online); this analysis did not include subcortical structures (such as the amygdala). In the first fully corrected test we found no regions that were correlated with the social network variables at conventional levels of statistical significance. In the second more exploratory analysis with a more lenient threshold $(p<.01$, uncorrected for multiple comparisons) we found that social network variables correlated significantly with the caudal inferior temporal sulcus (cITS), caudal superior frontal gyrus (cSFG), and subgenual anterior cingulate cortex ( $\mathrm{sgACC}$ ). Separate analyses of young and older participants demonstrated very consistent findings, supporting the reliability of these observations. For more details, see Supplementary Results, Supplementary Figure 1, and Supplementary Tables 2 and 3 online.

To our knowledge, these findings demonstrate the first link between amygdala volume and social network characteristics within a single species. Although our findings do not test an evolutionary hypothesis specifically, they, along with cross-species studies in nonhuman primates 7,15 , are consistent with the hypothesis that the primate amygdala evolved, in part, under the pressures of increasingly complex social life. In addition, that individuals with larger sgACC and cITS volumes also report larger and more complex social networks support the hypothesis that the amygdala expanded in conjunction with some other brain regions to which it is densely connected7. The correlation found for the cSFG requires further investigation. Results from the exploratory analysis should be taken as preliminary findings that could guide future work aimed at examining the distributed network of brain regions that might support social network size and complexity.

Humans are inherently social animals. We play, work, eat, and fight with one another. A larger amygdala might enable us to more effectively identify, learn about, and recognize socioemotional cues in conspecifics 4 , allowing us to develop complex strategies to cooperate and compete1. 


\section{Supplementary Material}

Refer to Web version on PubMed Central for supplementary material.

\section{Acknowledgments}

This study was supported by grants from the National Institute of Health Director's Pioneer Award (DP1OD003312) and the National Institute on Aging (R01 AG030311, R01 AG029411, and R21-AG29840). The authors thank Michael Brickhouse for assistance with morphometric analyses.

\section{References}

1. Silk JB. Social components of fitness in primate groups. Science. 2007; 317:1347-1351. [PubMed: 17823344]

2. Dunbar RIM. The social brain hypothesis. Evolutionary Anthropology. 1998; 6:178-190.

3. Buchanan, TW.; Tranel, D.; Adolphs, R. The human amygdala in social function. In: Whalen, PJ.; Phelps, EA., editors. The human amygdala. The Guilford Press; New York: 2009. p. 289-318.

4. Adolphs R. The social brain: neural basis of social knowledge. Annu Rev Psychol. 2009; 60:693716. [PubMed: 18771388]

5. Barton RA. Visual specialization and brain evolution in primates. Proc Biol Sci. 1998; 265:19331937. [PubMed: 9821360]

6. Barton, RA.; Dunbar, RIM. Evolution of the social brain. In: Byrne, R.; Whiten, A., editors. Machiavellian Intelligence II. Cambridge University Press; 1997. p. 240-263.

7. Barton, RA.; Aggleton, JP. Primate evolution and the amygdala. In: Aggleton, JP., editor. The amygdala: a functional analysis. 2000. p. 480-508.

8. Lewis KP, Barton RA. Amygdala size and hypothalamus size predict social play frequency in nonhuman primates: a comparative analysis using independent contrasts. J Comp Psychol. 2006; 120:31-37. [PubMed: 16551162]

9. Cohen S, Doyle WJ, Skoner DP, Rabin BS, Gwaltney JM Jr. Social ties and susceptibility to the common cold. JAMA. 1997; 277:1940-1944. [PubMed: 9200634]

10. Wright CI, et al. Neuroanatomical correlates of extraversion and neuroticism. Cereb Cortex. 2006; 16:1809-1819. [PubMed: 16421327]

11. O'Brien LM, et al. Adjustment for whole brain and cranial size in volumetric brain studies: a review of common adjustment factors and statistical methods. Harv Rev Psychiatry. 2006; 14:141151. [PubMed: 16787886]

12. Russell D, Cutrona CE, Rose J, Yurko K. Social and emotional loneliness: an examination of Weiss's typology of loneliness. J Pers Soc Psychol. 1984; 46:1313-1321. [PubMed: 6737214]

13. Gurung RAR, Taylor SE, Seeman TE. Accounting for changes in social support among married older adults: Insights from the MacArthur studies of successful aging. Psychology and Aging. 2003; 18:487-496. [PubMed: 14518810]

14. Diener E, Emmons RA, Larsen RJ, Griffin S. The Satisfaction with Life Scale. Journal of Personality Assessment. 1985; 49:71-75. [PubMed: 16367493]

15. Barger N, Stefanacci L, Semendeferi K. A comparative volumetric analysis of the amygdaloid complex and basolateral division in the human and ape brain. Am J Phys Anthropol. 2007; 134:392-403. [PubMed: 17661399] 
A

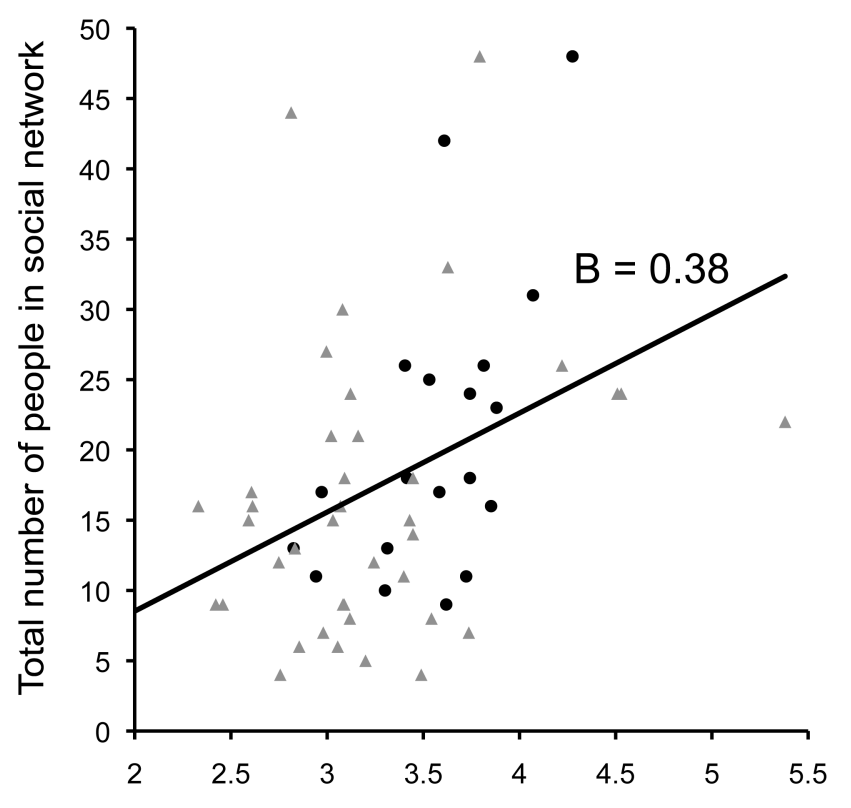

B

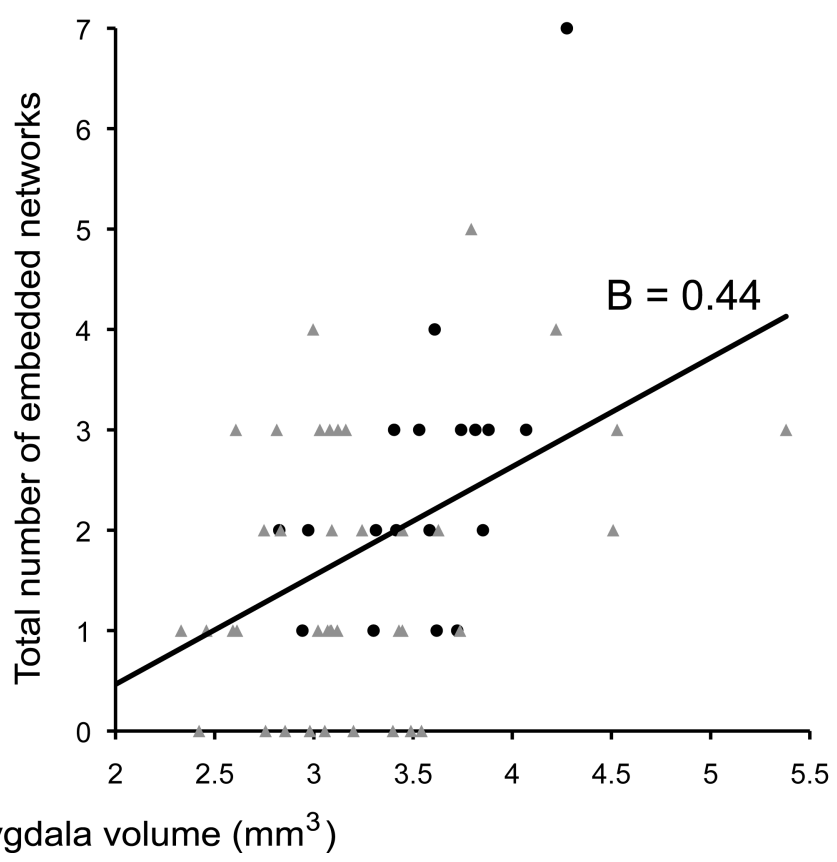

Fig. 1.

Amygdala volume correlates with social network size and complexity. Panels A and B plot social network variables ( $\mathrm{Y}$-axis) against total adjusted amygdala volume (X-axis). Data points from young participants are in black circles and older participants in grey triangles. A line of best fit with standardized regression coefficients (B) are also displayed for the entire sample. 


\section{Table 1}

Linear regressions using amygdala and hippocampal volumes (corrected for total intracranial volume) as independent variables and social network characteristics as dependent variables for the whole group as well as the young, older, male, and female subgroups.

\begin{tabular}{|c|c|c|c|c|}
\hline & \multicolumn{2}{|c|}{ Amygdala } & \multicolumn{2}{|c|}{ Hippocampus } \\
\hline & Left & Right & Left & Right \\
\hline \multicolumn{5}{|l|}{ Whole group $(n=58)$} \\
\hline Social Network Size & $0.38,2.84(0.006)$ & $0.29,2.15(0.036)$ & $0.23,1.66(0.103)$ & $0.10,0.72(0.472)$ \\
\hline Social Network Complexity & $0.39,3.13(0.003)$ & $0.30,2.32(0.024)$ & $0.25,1.89(0.064)$ & $0.15,1.08(0.286)$ \\
\hline \multicolumn{5}{|l|}{ Young group $(n=19)$} \\
\hline Social Network Size & $0.58,2.96(0.009)$ & $0.54,2.61(0.018)$ & $0.22,0.94(0.359)$ & $-0.07,-0.27(0.792)$ \\
\hline Social Network Complexity & $0.56,2.81(0.012)$ & $0.57,2.85(0.011)$ & $0.22,0.94(0.360)$ & $-0.11,-0.45(0.656)$ \\
\hline \multicolumn{5}{|l|}{ Older group $(n=35)$} \\
\hline Social Network Size & $0.32,2.05(0.048)$ & $0.24,1.52(0.138)$ & $0.27,1.68(0.102)$ & $0.18,1.11(0.274)$ \\
\hline Social Network Complexity & $0.38,2.50(0.017)$ & $0.28,1.76(0.086)$ & $0.32,2.06(0.047)$ & $0.27,1.69(0.099)$ \\
\hline \multicolumn{5}{|l|}{ Males $(n=36)$} \\
\hline Social Network Size & $0.31,1.87(0.07)$ & $0.18,1.06(0.298)$ & $0.19,1.15(0.259)$ & $0.07,0.38(0.706)$ \\
\hline Social Network Complexity & $0.43,2.79(0.009)$ & $0.27,1.60(0.118)$ & $0.35,2.19(0.036)$ & $0.22,1.23(0.203)$ \\
\hline \multicolumn{5}{|l|}{ Females $(n=22)$} \\
\hline Social Network Size & $0.52,2.72(0.013)$ & $0.62,3.53(0.002)$ & $0.20,0.92(0.367)$ & $0.22,1.00(0.329)$ \\
\hline Social Network Complexity & $0.45,2.27(0.034)$ & $0.60,3.39(0.003)$ & $0.14,0.64(0.529)$ & $0.20,0.91(0.372)$ \\
\hline
\end{tabular}

For the whole-group analysis, we controlled for age. The table displays standardized regression coefficients (B), $t$ values, and $p$ values ( 2 tailed, in parentheses). 